\title{
Adenocarcinoma endometrioide de ovário em parede abdominal
}

\author{
Endometrioid adenocarcinoma of ovary in abdominal wall
}

Manlio Basilio Speranzini', Sérgio Pezzolo', Rodrigo Bornstein Martinelli', Liliana de Souza e Silva Ducatti', Marcus Paulo Lemos Lemes', Marita Von Rautenfeld', Gilvane Souza Honório', Rodrigo Castro'

\section{Resumo}

Introdução: A transformação maligna da endometriose pode ocorrer em 0,7-1\% das pacientes e 78,7\% desses casos ocorrem nos ovários. Este trabalho relatou o caso de um adenocarcinoma endometrioide de ovário em parede abdominal após dois partos cesarianos. Relato de caso: Paciente de 52 anos apresentava dor bem localizada em região de hipogástrio com presença de tumoração no mesmo local há 1 ano. A referida dor piorava no período menstrual. Relatou, ainda, aumento do número de ciclos menstruais (duas vezes ao mês). A realização de exames de imagem evidenciou formação nodular heterogênea medindo 45 × 45 mm, subjacente à cicatriz cirúrgica na extremidade direita (pfannestiel), com ausência de linfoadenopatias. Foi realizada a exérese do tumor da parede abdominal, que revelou, após exame anatomopatológico, ser um adenocarcinoma endometrioide de ovário. Discussão: A manipulação cirúrgica da cavidade pélvica pode ser considerada um fator de risco no desenvolvimento da endometriose extragonadal. Além disso, o hiperestrogenismo, seja endógeno, seja exógeno, tem sido considerado como um fator de risco no desenvolvimento do câncer originado da endometriose. A malignização extragonadal da endometriose deve ser considerada como um diagnóstico diferencial em qualquer mulher com massa em parede abdominal, dor pélvica e/ou abdominal, uso de terapia de reposição hormonal, histórico de manipulação cirúrgica da cavidade pélvica e sangramentos vaginais.

Palavras-chave: Carcinoma endometrioide; ovário; endometriose; parede abdominal.

\section{Abstract}

Introduction: The malignant transformation of endometriosis can occur in $0.7-1 \%$ of the patients and $78.7 \%$ of these cases occur in the ovaries. This paper reported a case of endometrioid adenocarcinoma of ovary in the abdominal wall after two cesarean deliveries. Case report: A 52-year patient presented well-localized pain at the hypogastrium region where a tumor has been present for 1 year. The related pain worsened during menstruation. She also related increase in the number of menstrual cycles (twice a month). The performance of imaging tests revealed heterogeneous nodular formation measuring $45 \times 45 \mathrm{~mm}$, behind the surgical scar on the right end (pfannestiel), with absence of lymphadenopathies. The excision of the tumor from the abdominal wall was performed and revealed, after anatomopathological examination, to be an endometriod adenocarcinoma of ovary. Discussion: The surgical manipulation of the pelvic cavity can be considered a risk factor in the development of extra-gonadal endometriosis. Moreover, the hyperestrogenic, either endogenous or exogenous, has been considered a risk factor in cancer development originated from endometriosis. The extra-gonadal malignancy of endometriosis should be considered as a differential diagnostic in any woman with mass in the abdominal wall, with pelvic and/or abdominal pain, hormonal replacement therapy, history of surgical manipulation of the pelvic cavity and vaginal bleeding.

Keywords: Carcinoma, endometrioid; ovary; endometriosis; abdominal wall.

Recebido: 4/3/2009

Revisado: $15 / 6 / 2009$

Aprovado: $13 / 1 / 2010$

\footnotetext{
Trabalho realizado no Hospital Estadual Mário Covas, Santo André (SP), Brasil

Serviço de Cirurgia do Aparelho Digestivo do Hospital Estadual Mário Covas da Faculdade de Medicina do ABC (FMABC), Santo André (SP), Brasil Endereço para correspondência: Rodrigo Castro - Avenida Lauro Gomes, 2.000 - CEP 09060-870 - Santo André (SP), Brasil - E-mail: rodrigocastroabc@gmail.com
} 


\section{Introdução}

Endometriose é uma doença benigna, que acomete as mulheres em idade reprodutiva e consiste na presença de estroma e glândulas endometriais em locais fora do útero, principalmente no ovário e no peritônio ${ }^{1}$. Porém, também pode ser encontrada no fundo de saco de Douglas (atrás do útero), no septo retovaginal (tecido entre a vagina e o reto), nas trompas de Falópio, na superfície do reto, nos ligamentos do útero, na bexiga e na parede da pelve ${ }^{2}$.

A prevalência da endometriose em mulheres entre 25 e 40 anos é estimada em 10\%, enquanto que essa patologia é encontrada em apenas $2-4 \%$ de mulheres menopausadas ${ }^{3,4}$. Acomete mais de $50 \%$ de mulheres inférteis e possui maior frequência em nulíparas ${ }^{5}$.

Os sintomas mais comuns da endometriose são:

- dor antes e durante o período menstrual, geralmente mais intensa do que as dores menstruais normais;

- dor durante e após a relação sexual;

- dor ao urinar e a evacuar;

- dor pélvica crônica;

- $\quad$ alterações na duração e no fluxo menstrual;

- infertilidade.

A suspeita do diagnóstico é clínica, a partir da queixa da paciente, sendo confirmado em associação com exame físico, que pode evidenciar massas e sensibilidade dolorosa à palpação. A videolaparoscopia é o padrão-ouro no diagnóstico de endometriose, devido à visualização direta da lesão, com a possibilidade de biópsia da mesma. A dosagem da concentração sérica do marcador tumoral CA-125 também é utilizado no diagnóstico. Porém, apresenta baixa sensibilidade e especificidade para o diagnóstico ${ }^{6}$.

Existem múltiplos mecanismos pelos quais a endometriose pode acontecer. Recentemente, Nisolle e Donnez sugeriram que a endometriose peritoneal, ovariana e no septo retovaginal possuem cada uma um mecanismo patológico diferente. Os fatores patológicos dessa doença incluem predisposição familiar, fatores imunológicos, fatores de adesão de células, fatores da angiogênese e fatores hormonais, como, por exemplo, o estrógeno. Este importante hormônio feminino é extremamente mitogênico do endometrioma e da endometriose ${ }^{7}$. Atualmente, existem evidências substanciais de que tanto a produção (hiperestrogenismo) quanto o metabolismo desse hormônio podem estar alterados na endometriose. Várias teorias são propostas, como a expressão anormal de enzima aromatase, que converte androgênios em estrogênios. A atividade da aromatase anormal, no tecido endometrial, é potencializada pela prostaglandina $E_{2}$, que estimula a produção contínua de estrogênio, aumentando sua concentração. Além disso, as pacientes com endometriose apresentam também a ausência da enzima 17-beta-hidroxiesteroide desidrogenase tipo 2 (17 $\beta$-HSD), que atua na conversão do estradiol em estrona, um estrogênio mais fraco ${ }^{7}$.
A ausência dessa enzima combinada com a alta concentração estrogênica pode ajudar a estabelecer e a estimular a doença. Por outro lado, a progesterona inibe a ação mitogênica do estrógeno, porém possui um efeito muito menor no tecido endometrioide do que no endométrio, quando comparado com o estrógeno ${ }^{8}$.

O adenocarcinoma endometrioide de ovário (ACEO) é o terceiro tipo de tumor maligno epitelial ovariano, depois do tipo seroso e mucinoso 9 . De todos os casos, apenas $28 \%$ são bilaterais. Os riscos do ACEO são relativamente mais altos em mulheres na quinta e sexta décadas de vida, embora, na literatura, existam casos descritos em mulheres mais velhas ${ }^{10}$.

A transformação maligna de endometriose para o ACEO é relatada desde $1925^{11}$, sendo descritos apenas 200 casos na literatura ${ }^{12}$. Sampson foi o primeiro que descreveu essa modificação, criando critérios para o estabelecimento da transformação maligna da endometriose: (1) deve existir endometriose na vizinhança do tumor; (2) a histologia do câncer necessita ter origem endometrial e (3) nenhum outro foco primário desse tumor pode ser encontrado. De acordo com esses critérios, a modificação de endometriose para ACEO ocorre em 0,7-1\% das pacientes, com $78,7 \%$ dos casos ocorrendo no ovário ${ }^{13}$.

\section{Relato de caso}

Paciente de 52 anos, branca, chegou ao nosso serviço referindo a presença de uma massa em fossa ilíaca direita há 1 ano, medindo em torno de $10 \mathrm{~cm}$, bem localizada, dolorosa à palpação e aos esforços. Referiu dor de tipo queimação, que piorava no período menstrual e melhorava somente com medicamentos (Feldene ${ }^{\circledR}$ supositório - sic). Foi encaminhada ao serviço de Ginecologia, com as mesmas queixas, onde se fez a hipótese diagnóstica de endometriose.

Questionada sobre o ciclo menstrual, a paciente referiu que, após o aparecimento da massa, o mesmo modificou-se, aumentando para dois ciclos mensais com aumento da secreção menstrual.

Com relação a antecendentes obstétricos, ela relatou duas gestações com partos cesáreos e, com relação a antecedentes cirúrgicos, colecistectomia há nove anos. Apresentou o uso de Feldene ${ }^{\circledR}$ supositórios há um ano (desde o aparecimento da massa) e remédio para emagrecer, suspenso na última semana.

Quando questionada sobre comorbidades na sua família, a paciente referiu que sua mãe havia falecido há 20 anos, devido a neoplasia de útero, não sabendo referir tipo histológico.

Ao exame físico (EF), notou-se presença de massa medindo, aproximadamente, $10 \mathrm{~cm}$ em fossa ilíaca direita, visível à inspeção, de consistência firme, não-móvel, dolorosa à palpação superficial e profunda. Ao restante do exame, nada digno de nota.

Foram colhidos exames laboratoriais e hormonais, os quais evidenciaram valor aumentado de estradiol e de progesterona (610 U/L e $29 \mathrm{mg} / \mathrm{mL}$, respectivamente). Foi solicitada ultrassonografia (US) abdominal e transvaginal. Na US abdominal, evidenciou-se uma for- 
mação nodular heterogênea medindo 45 x 45 mm, subjacente à cicatriz cirúrgica na extremidade direita (pfannesteal), com ausência de linfoadenopatias e vascularização regional preservada.

Apresentando esse quadro clínico e os resultados dos exames de imagens descritos acima, foi proposta a cirurgia de exérese de tumor de parede abdominal com abdominoplastia.

Com incisão abdominal infraumbilical sobre cicatriz prévia, foi visualizada uma massa de aspecto lobulado, com vários cistos de contéudo escuro, não-espesso e sem odor. Iniciou-se a dissecção do tumor da parede que se estendia da sínfise púbica até a linha arqueada esquerda, ultrapassando a linha alta por $5 \mathrm{~cm}$ contralateral. Finalizada a dissecção, o próximo passo foi a revisão da hemostasia e ligação dos vasos de maiores calibres com algodão 3-0. Foi realizada a dissecção da aponeurose e da musculatura adjacente, com aproximação da musculatura lateral com vycril 2-0 em pontos separados em "U”.

Após a lavagem da ferida operatória, colocou-se a tela de Marlex ${ }^{\mathbb{R}}$ em falha de aponeurose com fixação com prolene 2-0; colocação de dreno portovac saindo pelas extremidades laterais da incisão. Finalizou-se o procedimento cirúrgico com o fechamento de subcutâneo e da pele e colocação de curativo oclusivo.

A massa tumoral (Figura 1) foi enviada para exame anatomopatológico, o qual revelou tratar-se de um adenocarcinoma endometrioide papilífero grau histológico II de ovário, estádio patológico IIIb.

A paciente evoluiu bem, sem nenhuma complicação pós-operatória. Foi encaminhada para a Oncologia para continuar com o tratamento quimioterápico.

\section{Discussão}

Embora a endometriose ocorra frequentemente nos órgãos pélvicos, em especial o ovário, é raro encontrar implantes distantes como os pulmões, nódulos linfáticos, pele e trato urinário ( $1 \%$ de todas as lesões endometrióticas $)^{14}$. O tecido endometrial ectópico pode extrinsecamente envolver ou intrinsecamente invadir a bexiga e/ou os ureteres e (menos comum) invadir a uretra e os rins, causando redução da função renal no momento do diagnóstico. Os sinais e sintomas mais comuns são dor abdominal e/ou pélvica, presença de massa pélvica e sangramentos vaginais ${ }^{15}$.

Hiperestrogenismo, seja endógeno, seja exógeno, tem sido considerado como um fator de risco no desenvolvimento do câncer derivado da endometriose. Zanetta et al. referiram em seu estudo que, quando a obesidade e o uso de estrógenos exógenos estão presentes concomitantemente, os riscos de desenvolvimento desse tipo de câncer aumentam $80 \%{ }^{16}$.
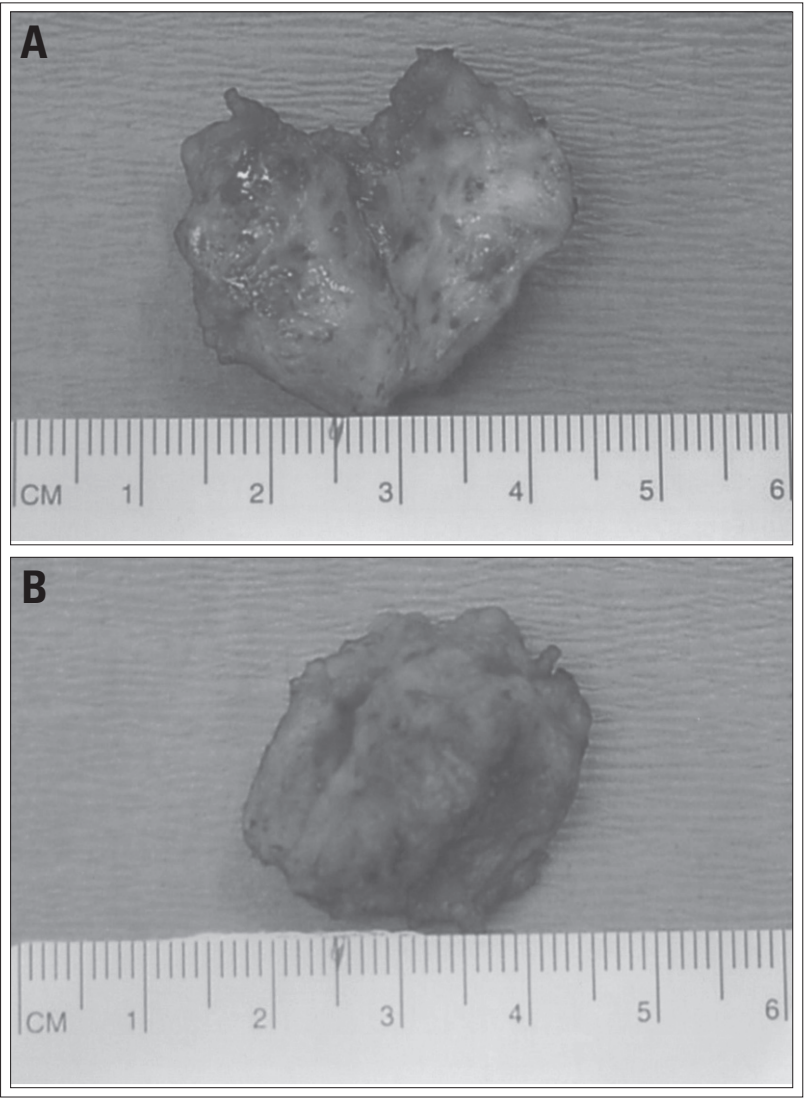

Figura 1 - Adenocarcinoma endometrioide papilífero. (A) Face anterior; (B) face posterior.

A manipulação cirúrgica da cavidade pélvica, como a histerectomia e o parto cesariano, é considerada um fator de risco no desenvolvimento da endometriose. Como visto no nosso caso, a paciente sofreu duas intervenções cirúrgicas na cavidade pélvica.

Embora a terapia de reposição hormonal não esteja inteiramente descrita na gênese do câncer em pacientes com endometriose, é bem mais provável que esteja relacionada a uma terapia adversa à do estrógeno $^{16}$. Nesse processo, acredita-se que o estrógeno não aja como um indutor carcinogênico, mas como um promotor da carcinogênese. A malignação associada com o hiperestrogenismo tem sido associada com adenocarcinomas bem diferenciados (69\%), presença de diferenciação escamótica (70\%) e sobrevivência em 5 anos de $82 \%{ }^{11}$.

Em conclusão, mesmo incomum, a malignação extragonadal da endometriose deve ser considerada como um diagnóstico diferencial em qualquer mulher com massa pélvica, com dor pélvica e/ou abdominal, uso de terapia de reposição hormonal, histórico de processos cirúrgicos pélvicos e sangramentos vaginais. É necessário um estudo detalhado da extensão da lesão pelo médico, com possível implantação em outros órgãos pélvicos. 


\section{Referências}

1. Clement PB. Diseases of the peritoneum. In: Kurman RJ, editor. Blaustein's pathology of the female genital tract. $5^{\text {th }}$ ed. New York: Springer-Verlag; 2002. p. 729-89.

2. Gücer F, Pieber D, Arikan MG. Malignancy arising in extraovarian endometriosis during estrogen stimulation. Eur J Gynaecol Oncol. 1998;19(1):39-41.

3. Olive DL, Schwartz LB. Endometriosis. N Engl J Med 1993;328(24):1759-69.

4. Punnonen R, Klemi PJ, Nikkanen V. Postmenopausal endometriosis. Eur J Obstet Gynecol Reprod Biol 1980;11(3):195-200.

5. Peterson EP, Behrman SJ. Laparoscopic of infertile patient. J Obstet Gynecol. 1970;36(3):363-7.

6. Ferreira CA, Camargo AF. Avaliação da dosagem do CA-125 sérico no diagnóstico da endometriose. J Bras Ginecol. 1994;104(9):311-4.

7. D'Hooghe TM, Hill JA. Endometriose. In: Berek JS, Adashi EY, Hillard PA Novak Tratado de Ginecologia. $12^{\text {th }}$ ed. Rio de Janeiro: Guanabara Koogan; 1998. p.628-48.

8. Wells M. Recent advances in endometriosis with emphasis on pathogenesis, molecular pathology, and neoplastic transformation. Int J Gynecol Pathol. 2004;23(4):316-20.

9. Tagashira Y, Shimada M, Kigawa J, Iba T, Terakawa N. Ovarian endometrioid adenocarcinoma arising from endometriosis in a young woman. Gynecol Oncol. 2003:91(3):643-7.
10. Russell P. Surface epithelial stromal tumors of the ovary. In: Kurman RJ, editor. Blaustein's pathology of the female genital tract. $5^{\text {th }}$ ed. New York: Springer-Verlag; 2002. p. 705-82.

11. Heaps JM, Nieberg RK, Berek JS. Malignant neoplasms arising in endometriosis. Obstet Gynecol. 1990;75(6):1023-8.

12. Salerno MG, Masciullo V, Naldini A, Zannoni GF, Vellone V, Scambia G. Endometrioid adenocarcinoma with squamous differentiation arising from ureteral endometriosis in a patient with no history of gonadal endometriosis. Gynecol Oncol. 2005;99(3):749-52.

13. Nishida M, Watanabe K, Sato N, Ichikawa Y. Malignant transformation of ovarian endometriosis. Gynecol Obstet Invest. 2000;50 Suppl 1: 18-25.

14. Brooks JJ, Wheeler JE. Malignancy arising in extragonadal endometriosis: a case report and summary of the world literature. Cancer. 1977;40(6): 3065-73.

15. Yantiss RK, Clement PB, Young RH. Neoplastic and preneoplastic changes in gastrointestinal endometriosis: a study of 17 cases. Am J Surg Pathol. 2000;24:513-24

16. Zanetta GM, Webb MJ, Li H, Keeney GL. Hyperestrogenism: a relevant risk factor for the development of cancer from endometriosis. Gynecol Oncol. 2000;79(1):18-22. 